\section{Inspection of Electrical Wires for Insulation Faults and Current Surges using Sliding Temperature Sensor based on Optical Microfiber Coil Resonator}

\section{G. Y. Chen, G. Brambilla and T. P. Newson}

We present a compact and robust temperature sensor based on an optical microfiber coil resonator packaged around a Teflon tube. The probe can efficiently slide along electrical wires to map the local temperature for locating insulation faults and reporting high current surges. A temperature responsivity of $95 \mathrm{pm} /{ }^{\circ} \mathrm{C}$ up to $\sim 80{ }^{\circ} \mathrm{C}$ was demonstrated. This sensor is potentially low cost and simple to fabricate, making it attractive for domestic and industrial applications.

Introduction: The main causes of overheating that lead to electrical fires in domestic and industrial environments are excessive current, poor connections and insulation breakdown. A variety of optical thermometers have been reported in recent years [1-7]. However, most devices require high precision in the placement and orientation of the sensor head for accurate results. Optical sensors fabricated from tapered optical fibers offer several potential benefits in comparison to conventional integrated micro-photonic devices, such as low insertion loss, inherent fiber compatibility and flexibility. Optical microfiber loop/coil resonators (MLR/MCR) assembled from self-coupled optical microfibers $(\mathrm{OM})$ have shown temperature sensing abilities [5-7]. However, these devices were always immobilized on a slab or support rod. In this Letter, we present a wire-mounted sliding Teflon tube coiled with MCR. Sliding probes can be used for rapid inspection of long electric wires for fault location. The integrated MCR maps the local temperature to identify positions with insulation faults that can result in electrical arcing. Defects in the insulation integrity can be distinguished by an increase in heat signature. The ring-shaped detection area facilitates rotationally symmetric coverage and thus removes the need for radial alignment of the sensor head. Additionally, upsurges in temperature generated by intense currents can also be identified. The minimum bend radii of OMs [8] allow a wide range of wire diameters to be probed, from several millimeters down to tens of micrometers.

Sensor Design and Working Principle: The 3D schematic of the temperature probe is shown in Fig. 1, which consists of a MCR wrapped around a hollow Teflon cylinder. The device can slide along electrical wires to measure the local temperature. Due to the electrical resistance of the current-carrying wire, high current will result in considerable heat dissipation. This heat is then transferred to the Teflon tube and the coiled OM via thermal conduction. Teflon (DuPont Teflon AF, $n=1.3$ ) was chosen for the tube material due its high melting point and chemical resistance. The effective index seen by the propagating mode in the OM and the physical length of each turn in the MCR will be modified by thermal expansion and the thermal-optic effect, leading to a shift in the resonant wavelength [7].

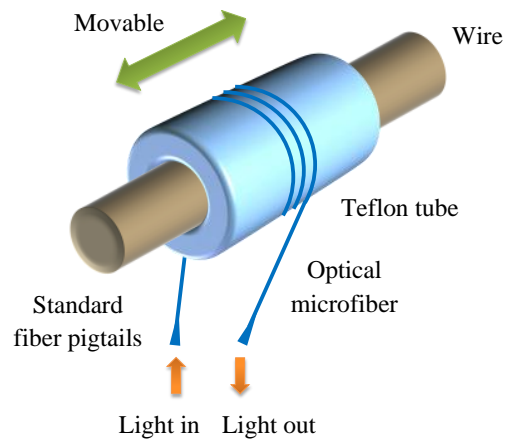

Fig. $13 D$ illustration of the MCR temperature probe capable of sliding along an electrical wire to measure the local temperature.

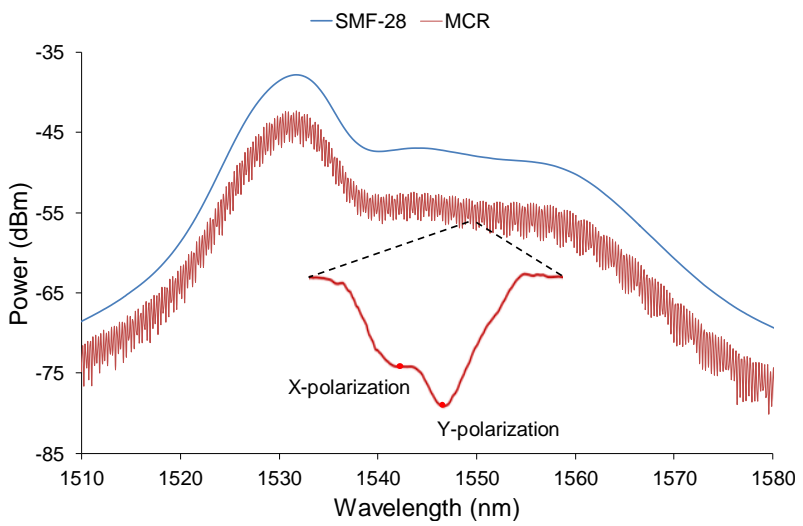

Fig. 2 Transmission spectrum of the packaged MCR and the original SMF-28. Inset: individual pair of resonances associated with the orthogonal polarizations arising from linear birefringence in the MCR.

Sensor Fabrication and Characterization: The first stage involves the fabrication of the sensor head. A $2 \mu \mathrm{m}$ diameter, $15 \mathrm{~mm}$ long OM was tapered using a pair of automated translation stages and a stationary ceramic micro-heater [9]. The tapering process lasted $\sim 10 \mathrm{~min}$ with an initial stretching speed of $1 \mathrm{~mm} / \mathrm{sec}$ that was gradually slowed to ensure an adiabatic taper profile for suppressing the excitation of higher order modes. The uniform diameter region of the taper was then carefully coiled 2.5 turns around a Teflon tube of 1.8/1 mm outer/inner diameter. A small winding pitch was chosen to enable coupling between adjacent OM turns, forming a MCR. A thin layer of U.V. polymer (Efiron UV$373, n=1.373$ ) was deposited on the surface of the Teflon tube before and after coiling to provide good optical confinement and geometric stability when cured. For the purpose of demonstration, a relatively long segment of Teflon tube was employed for ease of handling during the OM winding process. The standard fiber pigtails of the MCR were also embedded in the same polymer coating on a glass slide and U.V. cured for robust packaging.

Characterization of the MCR was performed by exciting the MCR with amplified spontaneous emission (ASE) from an Erbium-doped fiber amplifier (EDFA). The transmission spectrum from Fig. 2 was captured using an optical spectrum analyser (OSA). The maximum resonance extinction ratio and insertion loss at the wavelength of $\lambda=$ $1550 \mathrm{~nm}$ were $\sim 3 \mathrm{~dB}$ and $\sim 5 \mathrm{~dB}$ respectively.

For testing the temperature sensor, a $0.34 \mathrm{~mm}$ diameter, $30 \mathrm{~mm}$ length of Nichrome wire was chosen as the heating element due to its large electrical resistance $(0.2 \Omega / \mathrm{cm})$ and thus thermal response to electrical current. The wire was inserted inside the MCR-coiled Teflon tube. Device accuracy was optimized though the use of thermal paste, which improved the conduction path between the Nichrome wire and the Teflon tube.

Experimental Setup, Results and Discussions: The optical setup of the MCR temperature sensor is shown in Fig. 3. Broadband light was provided by the EDFA. As the electrical current $(I)$ delivered by a tunable power supply passed through the Nichrome wire, power dissipated as heat was absorbed by the MCR and its surrounding polymer through thermal conduction. This changed the local temperature and caused a resonant wavelength shift of the MCR. The transmission spectrum was collected by the OSA as DC current was

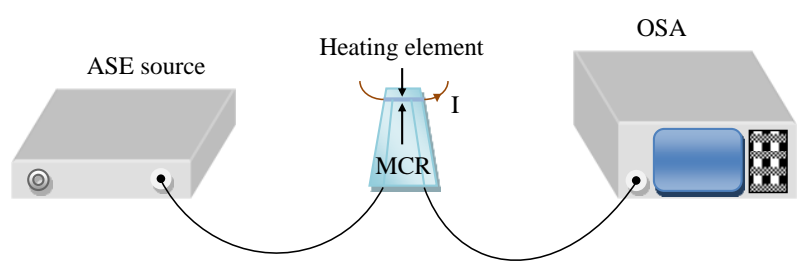

Fig. 3 Schematic of experimental setup for testing the sensor. 


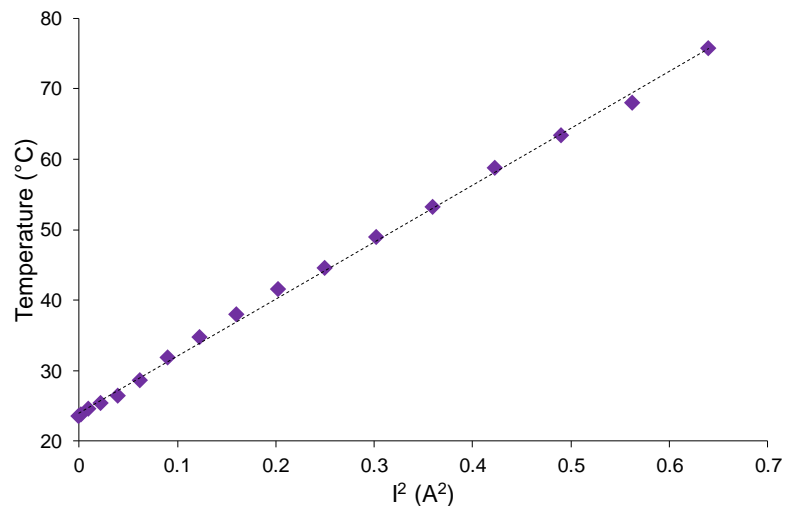

a

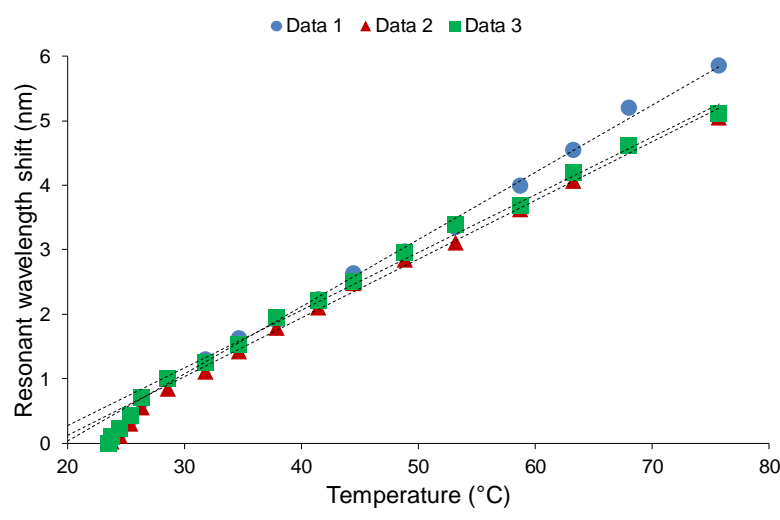

b

Fig. 4 Experimental results of the heating element characterization and the responsivity of the MCR temperature sensor.

a Thermal response of Nichrome wire.

$b$ Spectral shift of MCR with temperature.

incremented from 0 to $800 \mathrm{~mA}$ in steps of $50 \mathrm{~mA}$. A commercial thermocouple was used to provide a temperature reading. The sensor head of the thermocouple is very small and thus has a negligible effect on the local temperature. A room temperature of approximately $23{ }^{\circ} \mathrm{C}$ was maintained whilst the measurement process was repeated 3 times.

The response time of the MCR temperature sensor was less than $1 \mathrm{~s}$ and the resonant wavelength shift stabilizes after $\sim 7 \mathrm{~s}$. The resonance dip of the Y-polarization was easily tracked due to its higher extinction ratio. Fig. 4a shows that the local temperature change is proportional to the square of the electrical current applied across the Nichrome wire, at $80.7^{\circ} \mathrm{C} / \mathrm{A}^{2}$. Fig. $4 \mathrm{~b}$ shows an average responsivity of $95 \mathrm{pm} /{ }^{\circ} \mathrm{C}$ between 26-76 ${ }^{\circ} \mathrm{C}$ from 3 sets of measurements. Datasets 2 and 3 show good agreement, whereas dataset 1 deviates at higher temperatures. This could be attributed to a small but steady rise in the ambient temperature during the time-frame of the $1^{\text {st }}$ set of measurements. The temperature sensitivity of the MCR is $0.21{ }^{\circ} \mathrm{C}$ when using an OSA with a wavelength resolution of $20 \mathrm{pm}$. The upper limit of measurable temperature can be extended by replacing the polymer packaging with Teflon, which has a higher melting point. The spatial resolution is governed by the width of the MCR, which is typically tens of microns.

Conclusion: A compact and robust temperature sensor based on a MCR coiled around a Teflon tube has demonstrated a responsivity of 95 $\mathrm{pm} /{ }^{\circ} \mathrm{C}$ between $26-76{ }^{\circ} \mathrm{C}$. The sensor can be readily slide along electrical wires to examine the insulation integrity by measuring the local temperature. The thermal response of high current surges can also be identified by monitoring the resonant wavelength shift of the MCR.

G. Y. Chen, G. Brambilla and T. P. Newson (Optoelectronics Research Centre, University of Southampton, Southampton, SO17 1BJ, United Kingdom)

E-mail: gyc1g09@orc.soton.ac.uk

\section{References}

1. Ding, M., Wang, P., and Brambilla, G., 'A microfiber coupler tip thermometer', Opt. Express, 2012, 20, (5), pp. 5402-5408.

2. Qian, W., Zhao, C., Chan, C. C., Hu, Li., Li, T., Wong, W. C., Zu, P., and Dong, X., 'Temperature sensing based on ethanol-filled photonic crystal fiber modal interferometer', IEEE Sens. J., 2012, 12, (8), pp. 2593-2597.

3. Silva, S., Pachon, E. G. P., Franco, M. A. R., Hayashi, J. G., Malcata, F. X., Frazão, O., Jorge, P., and Cordeiro, C. M. B., 'Ultrahighsensitivity temperature fiber sensor based on multimode interference', Appl. Optics, 2012, 51, (16), pp. 3236-3242.

4. Ma, J., Ju, J., Jin, L., Jin, W., and Wang, D., 'Fiber-tip micro-cavity for temperature and transverse load sensing', Opt. Express, 2011, 19, (13), pp. 12418-12426.

5. Zeng, X., Wu, Y., Hou, C., Bai, J., and Yang, G., 'A temperature sensor based on optical microfiber knot resonator', Opt. Commun., 2009, 282, (18), pp. 3817-3819.

6. Wu, Y., Jia, L., Zhang, T., Rao, Y., and Gong, Y., 'Microscopic multi-point temperature sensing based on microfiber double-knot resonators', Opt. Commun., 2012, 285, (8), pp. 2218-2222.

7. Chen, G. Y., Lee, T., Jung, Y., Belal, M., Brambilla, G., Broderick, N. G. R., and Newson, T. P., CLEO/Europe, 2011, CH2_2.

8. Yu, H., Wang, S., Fu, J., Qiu, M., Li, Y., Gu, F., and Tong, L., 'Modeling bending losses of optical nanofibers or nanowires', Appl. Optics, 2009, 48, (22), pp. 4365-4369.

9. Brambilla, G., 'Optical fibre nanowires and microwires: a review', J. Opt., 2010, 12, (4), pp. 043001-043020. 